\title{
Éros et Thanatos, ou le rouge et le noir robbe-grillétiens
}

\author{
Christian Milat \\ Université d'Ottawa
}

La critique a depuis longtemps mis l'accent sur la violence sexuelle qui s'exerce de façon explicite, voire exhibitioniste, dans les romans d'Alain Robbe-Grillet : le sang coule et, parfois, la mort s'ensuit. Cette violence, qui s'exprime au travers de rapports de domination entretenus par l'homme à l'encontre de la femme, a donné lieu à de nombreux commentaires, relevant par exemple «les jeux sadiques d'un érotisme glacé » (Bonnefoy, 1978, p. 29) où «le fétichisme sexuel » (Dhaenens, 1970, p. 11) s'affiche ou encore caractérisant des textes où la féminité «est la proie ignorante d'instances phallocratiques » 
(Forrester, 1976, p.9) habitées par "un érotisme effréné et sadique» (Linkhorn, 1976, p. 37). Dès Le Voyeur, de vives réactions sont enregistrées. Ainsi, après que le feuilletoniste du Monde eut dénoncé le caractère "pathologique » (Henriot) du récit, dont l'auteur, tenu pour un assassin ou un fou, est voué aux tribunaux compétents en matière d'attentats aux mœurs ou à l'asile de Sainte-Anne, le héros du roman, auquel le romancier est parfois identifié, est considéré comme un obsessionnel mû par « la frayeur d'être aimé » (Anzieu, 1965, p. 637), un malade atteint «d'épilepsie » (Weil-Malherbe, 1965, p. 470), « un être aliéné par sa psychose » (Goulet, 1982, p. 6) ou « un obsédé [...] que la passion va pousser jusqu'au meurtre » (Stoltzfus, 1964, p. 164). Un roman sentimental, le dernier texte de Robbe-Grillet, publié en 2007, a été l'objet de recensions tout aussi acerbes. Ainsi, dans L'Express en ligne, Baptiste Liger le présente comme un « $[\mathrm{r}]$ oman ignoble » comportant « une série de scènes de barbarie nauséeuses [...] d'une cruauté et d'une amoralité absolues ». Dans leurs blogs, Raphaël Sorin traite Robbe-Grillet de «vieux dégueulasse » et Pierre Assouline déclare que la lecture de ce livre « écœurant » provoque «lassitude [...] ennui [...] accablement ». Jérôme Garcin, dans Le Nouvel Observateur, parle d'un "conte de fées, version Michel Fourniret», en référence au violeur de jeunes filles et tueur en série. Dans Lire, Frédéric Beigbeder qualifie «les crimes qui font fantasmer Robbe-Grillet (inceste, pédophilie, sadisme, séquestration, torture et meurtres d'enfants, etc.) » de "pornographie de gare ». Michel Contat lui-même, pourtant fin connaisseur et, d'ordinaire, admirateur éclairé des œuvres robbe-grillétiennes, déplore cette fois dans Le Monde des livres «les infortunes du pornographe»: dans le «catalogue complet de perversions sadiques et de tortures sexuelles » que constitue le roman, il ne 
perçoit que «les fantasmes sado-érotiques d'un vieil écrivain qui [...] force la note pour parvenir à ranimer en lui des désirs fléchissants ». Il faut avouer que ces critiques ne peuvent qu'avoir été confortées par un auteur qui, non sans une complaisance sans doute quelque peu suspecte, confie par exemple dans Le Miroir qui revient que "seules des mises en scène (ou des imaginations) "perverses" excitent [s]on désir, ce qui va d'autant moins sans problèmes qu'[il est] attiré surtout par les très jeunes filles » (p. 44).

Délaissant ces points de vue psychologisants, l'étude qui va suivre s'attachera, dans un premier temps, à montrer comment, chez Robbe-Grillet, le rouge et le noir, le sang et la mort se rejoignent par le biais de la violence érotique et, dans un second temps, à proposer, pour ces trois éléments, deux pistes d'interprétation.

\section{Éros et la violence masculine vis-à-vis de la femme}

La couleur rouge, c'est un truisme, correspond notamment à l'«image [...] d'Éros libre et triomphant» (Chevalier et Gherbrant, 1982, p. 832). Les relations entre cette couleur et la sexualité sont très nombreuses dans les romans de RobbeGrillet. Ainsi, dans Le Voyeur, la couleur rouge, associée au feu, évoque la brûlure du désir : «Une sorte de chaleur arrivait de la chambre [du cafetier], comme si un foyer quelconque y brûlait » $(V$, p. 68). Les «draperies d'un rouge sombre» (p. 68) de cette chambre renvoient aux «draperies rouges » (p. 45) du lit qui figure sur une affiche de cinéma et auxquelles est associée une scène à contenu érotique. En effet, le lit y présente « un aspect de lutte» (p.68). La femme, assure le narrateur, «aurait eu 
besoin d'une correction sévère» (p. 85), elle mériterait le «fouet » (p. 59 et 141), des « claques » (p. 59). D’où le « bruit de gifle» (p. 21, 75 et 78) qui, émis par les vagues, complète le geste ébauché par l'homme de la chambre de laquelle provient une plainte de femme : "Debout près du lit, légèrement penchée au-dessus, une silhouette masculine levait un bras vers le plafond. » (p. 28) L'homme, qui «immobilis[e] d'une seule main les deux poignets [de la jeune femme] derrière le dos » (p. 45), est doté d'une «stature colossale» (p. 45). C'est un « géant» (p. 78), un « bourreau » (p. 45) qu'affronte une « proie [...] tellement menue qu'elle [...] paraît presque disproportionnée» (p. 78). Elle, elle est une jeune femme aux cils «de poupée» (p. 56 et 78), à l'«allure lente et fragile de poupée articulée »(p.64). C'est cette même fragilité qui est mise en relief par le moelleux du sexe féminin, moelleux opposé à la rigidité de l'organe masculin, ainsi qu'il apparaît au travers de cette représentation de l'accouplement qui se masque sous l'illustration figurant sur une boîte de couteaux emballés dans un carton portant « un encadrement rouge » (p. 55) :

Le dessin en représentait un arbre au tronc svelte et rectiligne, terminé par deux branches en i grec qui portaient une petite touffe de feuillage - dépassant à peine les deux branches sur les côtés, mais retombant jusqu'au creux de la fourche. (p. 55)

D'autres figures du phallus, toujours de couleur rouge, sont encore perceptibles: un poisson «long comme un poignard» $(V, 70)$, « une anguille rouge, ou un barbet» (p. 177), « la pointe rouge » (p. 236) d'une cigarette.

Le rapport de force qui s'instaure ainsi entre les deux sexes se clôt finalement par une union qui prend la forme d'un viol. En effet, même si celui-ci n'est pas explicitement mentionné, plusieurs signes en autorisent la supposition: le 
prénom de la victime, Violette, bien évidemment, et aussi, par exemple, le sort réservé à un crabe par un personnage masculin : " avec la grande lame de son couteau il transperça le ventre » $(V$, p. 137-138), là « où la carapace blanchâtre dessinait une sorte d'i grec» (p. 144). En fait, l'affrontement de l'homme et de la femme débouche inévitablement sur la mort. Déjà annoncée, à titre proleptique, sur l'affiche de cinéma, par le geste de l'homme qui « serrait à la gorge » (p. 45) sa victime, la mort, une fois l'union consommée, frappe la jeune femme, sans doute étranglée - la cordelette recueillie par le personnage principal, Mathias, est dotée de «spires [...] serrées à l'étranglement » (p. 10) -, puis « poussée» (p. 177) dans la mer. Elle y rejoint la jeune vierge de la légende, « dévor[ée] vivante [...] sous l'œil du sacrificateur» (p. 221) par «un monstre gigantesque au corps de serpent» (p. 221), le serpent constituant le symbole bien connu « de la libido » (Chevalier et Gheerbrant, 1982, p. 868). Dans Le Voyeur, la mort apparaît en particulier dans la «robe noire » $(V, 171)$ que portent Violette et l'ensemble des autres personnages féminins qui ne sont qu'une manifestation particulière de l'enfant, et aussi dans la bouée, «d'une belle couleur noire » (p. 255), qui flotte dans la mer en suivant « le mouvement des vagues» (p. 255) comme le cadavre de la jeune fille flotte lui-même en fonction du «va-etvient des vagues » (p. 175).

Nous retrouvons d'autres exemples de la violence caractérisant les rapports entre les deux sexes dans Projet pour une révolution à New York. Éros y est là encore associé à la chaleur. Ainsi, le « supplice» $(P$, p. 98) de Joan a lieu dans un «studio surchauffé » (p. 96). De plus, l'ardeur du sexe masculin, déjà manifestée au travers des "crocs aigus, rougis au feu » (p.196), de l'araignée, transparaît également dans une 
«cigarette à moitié consumée» (p. 144), dans «le bout incandescent de [...] cigares» (p. 208) et dans le «fer [à repasser] brûlant » (p. 82) que Joan « laisse tomber [...] sur [s]a robe verte » (p. 82), cette robe grillée «laiss[ant] un large trou triangulaire à la place du sexe » (p. 82) préfigurant le « creux de la plaie rouge sombre qui remplace [son] pubis » (p. 213) et la blessure qui lui est infligée par son tortionnaire.

La domination exercée sur la femme se manifeste par le fréquent usage d'une "grosse corde» $(P$, p. 88), de «cordelettes» (p.10 et 192), « de cordes de chanvre» (p.143, voir p. 194) ou de " chaînes » (p.181 et 208), lesquelles permettent aux bras et à la poitrine d'être "emprisonn[és]» (p. 88), aux mains d'être « liées dans le dos » (p. 88) et aux chevilles d'être «attachées» (p. 88, voir p. 8). Ainsi «ligotée» (p. 87), « immobilis[ée]» (p.17 et 170), la femme devient une «prisonnière » (p.9, 88, 92, 142, 150, 171, 181, 194, 192 et 210), une « captive » (p.94, 150, 170 et 202), une « esclave craintive» (p. 70) qui «ressen[t] son impuissance» (p. 171), « abandonn[e] toute velléité de résistance » (p. 171) et « obéi[t] [...] bien sagement» (p. 171) aux ordres de "son seigneur» (p. 70) et maître. Parfois, pour disposer d'une «chair sans défense » (p. 171), l'homme réduit la femme à l'inconscience au moyen d'un «tampon imbibé d'éther» (p.9) ou d'une « substance narcotique » (p. 89) quelconque.

Rendu « docile » $(P$, p. 10, 70 et 181) par la drogue ou par les «gifles» (p.27), «deven[u] malléable comme une poupée de chiffon » (p. 19), le corps de la femme est pénétré, d'où des déchirures (voir p.181, 184 et 191) ou des mutilations (voir p.182, 185, 188 et 213) qui se traduisent par l'écoulement de «filets de sang» (p. 182, voir p. 49, 99, 142 et 211) se 
transformant bientôt en "une petite mare » (p. 185), puis en « une mare de sang» (p. 213), «liquide visqueux» (p. 125), « rouge vif, épais » (p.124 et 138).

Ainsi «torturée» (p. 99, voir p. 152, 181 et 201), la femme «se tord au paroxysme de la souffrance» (p. 180), poussant un « cri horrible » (p. 194), un « long cri de souffrance et de terreur» (p.9), de «longs hurlements de douleur» (p. 142). Les suppliciées éprouvant alors

une série de spasmes sexuels de plus en plus forts et prolongés, devenant vite extraordinairement douloureux, se terminant au bout de plusieurs heures par la mort du sujet dans les convulsions combinées de la jouissance la plus vive et des plus atroces souffrances (p. 202).

Pareillement, après l'amour, Laura « sembl[e] morte » $(P$, p. 19). C'est la raison pour laquelle le sexe masculin est suggéré par «le canon» (p.97) d'une «mitraillette» (p. 82) et sa décharge évoquée par « une rafale de mitraillette » (p.68), à la suite de laquelle Laura « reste étendue sur le dos, de tout son long, bras et jambes écartés en croix» (p. 69). Mais il arrive également que la mort soit directement provoquée par le feu d'Éros, «l'incendie » (p. 153) constituant, à côté du « viol [...] [et de] l'assassinat » (p. 153), l'un des « trois actes métaphoriques » (p.153) préconisés par les adeptes de la «révolution»dont traite le roman. C'est pourquoi «le supplice du feu» (p.181) conduit le narrateur à « enflamme[r] le buisson roux » (p. 180), «le sexe imbibé d'essence» (p. 179) de Joan. 


\section{La femme comme métaphore}

Bien évidemment, et nombreux sont les critiques qui ne s'en sont pas privés, il est possible de lire les romans robbegrillétiens au premier degré et d'y déceler l'illustration, voire la promotion de pratiques sadomasochistes plus ou moins déviantes, plus ou moins perverses. Mais on peut les aborder différemment et tenter d'en tirer une herméneutique en proposant deux pistes d'interprétation possible.

La première sera celle de l'alchimie. La philosophie hermétique ${ }^{1}$ pose l'existence d'un « homme primordial, créé [...] mâle et femelle, c'est-à-dire androgyne » (Evola, 1988, p. 289), correspondant à un état pouvant être défini « comme celui d'un être absolu (non brisé, non "duel”) ». Tout désir comportant un mouvement vers autre chose que soi » (Evola, 1988, p. 171), c'est sous l'impulsion d'Éros qu'« Adam engendra d'abord Ève comme une image magique (nous pourrions dire : comme une image de fièvre projetée par son désir même) à laquelle il fournit ensuite une substance terrestre » (Evola, 1988, p. 291). C'est donc à cause d'Éros que l'unité se fragmente pour faire place à deux opposés disjoints. Or, l'alchimiste vise à transformer Éros, ce "poison[,] en remède ou "nectar", [employant ainsi] aux fins de la libération [l]es mêmes forces qui ont conduit ou qui peuvent conduire à la chute » (Evola, 1988, p. 315). En effet, l'androgyne une fois divisé, Éros nourrit, toujours en tant que désir de l'autre, «l'impulsion de reconstituer l'unité primordiale» (Evola, 1988, p. 68). C'est

\footnotetext{
${ }^{1}$ L'alchimie, dont les concepts sont volontairement voilés, est néanmoins plus ou moins explicitée par trois de ses adeptes - Canseliet (1972), Fulcanelli (1964 et 1965) et Valentin (1956) - ainsi que dans Alleau (1953), Eliade (1978), Evola (1967 et 1988), Guénon (1994) et Jung (1970 et 1971).
} 
pourquoi Éros est considéré par les alchimistes comme le «maître éternel de l'œEuvre » (Fulcanelli, 1930, t. II, p. 160). Or, s'il est vrai que le texte robbe-grillétien est marqué par la présence «à chaque instant $\mathrm{d}[\mathrm{e}]$ couples de contraires irréconciliables » (Robbe-Grillet, 1985, p. 116), il reste que ces opposés ne se font pas face de manière statique, mais qu'ils sont engagés dans un processus dynamique, fait d'attraction et de répulsion. En outre, dans tous les romans, c'est Éros qui œuvre au sein de ce processus, ainsi que le souligne par exemple Simon, le narrateur de Djinn: "La lutte des sexes [...] est le moteur de l'histoire. » (D, p. 19) Notons que ce narrateur porte un prénom évoquant l'unité hermétique, puisque Simon signifie


1930, t. I, p. 250), et qu'il est doté de plusieurs patronymes, Le/cœur (voir $D$, p. 7), Koer/shimen (voir p. 8), Kör/simos (voir p. 8) et Le/cœur/ovich (voir p.46), renfermant tous, littéralement ou phonétiquement, le lexème cœur, comme si Éros constituait l'agent conduisant à l'unité, personnifiée par l'« androgyne » (p. 13 et 133). De fait, tout comme, dans l'opus alchimique, c'est grâce à l'action d'Éros que les opposés se métamorphosent en suivant trois phases principales, manifestées par l'@Euvre au Noir, puis par l'CEuvre au Blanc et, enfin, par l'œEuvre au Rouge, de même l'ensemble des romans robbe-grillétiens est ponctué par ces trois stades (voir Milat, 2001), qui doivent pareillement leur succession aux effets du désir : «L'amour, ça fait faire de grandes choses. » (D, p. 49)

Dans Projet pour une révolution à New York, par exemple, la révolution, qui est au principe de la diégèse et dont le rouge est la couleur emblématique, ne vise rien d'autre que la reconstitution de l'unité. Au cours d'une « réunion» $(P$, p. 16 et 37) où les discours, remplis «de constantes [...] antithèses » 
(p. 37), font l'objet d'une «attention religieuse» (p. 40), l'organisation terroriste, «qui appartiendrait à l'église manichéiste unifiée » (p.56), y expose les propriétés de "la couleur rouge", envisagée comme solution radicale à l'irréductible antagonisme entre le noir et le blanc » (p. 38), formule qui, sortie d'un contexte alchimique, est dépourvue de sens. Les références à l'alchimie étant, dans Projet pour une révolution à New York comme dans les autres romans robbegrillétiens, innombrables, je me limiterai à deux exemples. Le premier a trait aux deux couleurs que les traités d'alchimie attribuent au sang, conseillant de «dissou[dre] et nourri[r] le vrai lion par le sang du lion vert. Car le sang fixe du lion rouge a été fait du sang non fixe du lion vert, parce qu'ils sont d'une seule nature. » (Valentin, 1956, p. 231) Or, JR, un personnage féminin de Projet, avant de baigner "dans une mare de sang » $(P$, p. 213), est présentée comme un «fauve» (p. 74); elle est dotée d' « yeux verts » (p. 74, 97, 176 et 181) et de « chaussures de cuir vert» (p. 78). Alors que le lion vert des alchimistes est également appelé "Émeraude des Philosophes» (Fulcanelli, 1926, p. 121), la robe de JR est de «couleur d'émeraude » $(P$, p.67). De plus, tandis que le même lion hermétique est aussi désigné par le «mercureau [...] ou petit mercure, qui est devenu notre maquereau, [...] poisson mystique [...] [devant] son nom et sa réputation à sa brillante coloration verte » (Fulcanelli, 1930, t. II, p. 248), la jeune femme est comparée à un «poisson des grandes profondeurs » $(P$, p. 67). Le second exemple porte sur la violence érotique faite aux femmes et sur la mort qui en découle. Ainsi, la femme de Projet est «torturée» (p. 99) comme, dans l'opus alchimique, le Mercure, qui correspond au principe féminin, subit le « cruciatus (supplice, torture) » (Jung, 1971, p. 153). De même, ce n'est pas un hasard si le 
"grésillement [...] dans les flammes [...] de la chevelure répandue, de la touffe de soie rousse » $(P$, p. 110), de Joan est associé à celui «du buisson ardent, ou de la toison d'or » (p.110). La jeune femme s'identifie ainsi à la vierge de l'alchimiste Melchior, laquelle a «été rendue mère par le feu sacré [...] [qu'elle a] porté comme le buisson ardent [...] [au profit d'un] bétail distingué par sa toison» (Szebeni, 1970, p. 506). Si, « [d] ans le langage des Adeptes, on appelle Toison d'or la matière préparée pour l'CEuvre, ainsi que le résultat final » (Fulcanelli, 1926, p. 194-195), force est de constater que «dans les rougeoiements de la torche vivante» $(P$, p. 180) qu'est Joan, c'est bien l'CEuvre au Rouge qui est atteint: «la troisième opération [...] donne la Pierre philosophale » (Fulcanelli, 1926, p. 203), c'est-à-dire la «pierre qui porte le signe du soleil » (Fulcanelli, 1930, t. I, p. 177). De fait, Joan, semblable au Mercure alchimique, a été transmutée jusqu'à rayonner « en un soleil flamboyant » $(P$, p. 213) et, comme dans le Grand OEuvre, cette transmutation s'effectue grâce au feu, grâce au feu d'Éros.

La seconde piste herméneutique a trait au couple d'opposés qu'il est possible de repérer derrière la dyade homme-femme. Confiant lui-même qu'il cherche dans son œuvre à " traduire » (Robbe-Grillet, 1984a, p. 41) son rapport «au monde et à [s]on être, [...] rapport où tout est double, contradictoire et fuyant», Robbe-Grillet oppose notamment "l'univers qu'affronte et sécrète tout à la fois notre inconscient» (1987, p.182) au "monde factice de la quotidienneté, celui de la vie dite consciente». Ces deux opposés renvoient à un autre couple, dont l'opposition est très fréquemment évoquée : il s'agit de la « lutte à mort de l'ordre et de la liberté. Ce conflit insoluble du classement rationnel et de 
la subversion, autrement nommée désordre. » (Robbe-Grillet, 1984a, p. 133) Le romancier est formel : «mes livres sont faits de la lutte entre l'ordre et le désordre » (1984b, p. 495). Cette lutte peut apparaître comme le conflit qui s'instaure chez le romancier au moment de l'écriture, dont Un roman sentimental, dernier roman de Robbe-Grillet, nous fournit plusieurs exemples ${ }^{2}$.

Enfant du narrateur, «Gigi » $(R S$, p. 14) - c'est le prénom de la jeune fille -, peut être considérée comme son texte ${ }^{3}$. Du reste, elle est présentée comme «sa créature » (p. 252), comme sa " propriété » (p. 206), et la séance de lecture qui s'engage entre le père et sa fille et au cours de laquelle celle-ci sera châtiée par celui-là - à coup de « cinglon[s] sur les fesses » (p. 15) - apparaît comme une séance de travail où le texte est relu pour être corrigé par l'écrivain. Robbe-Grillet, qui amende son texte après l'avoir relu «pendant plusieurs jours à haute voix » (Robbe-Grillet, 1976, p.428), est en effet ce père qui "surveille sans complaisance [sa fille], s'apprêtant à châtier d'un coup sec de sa badine la moindre faute de texte, de rythme» $(R S$, p. 14). Ces séances de lecture permettent à la fille-texte «de se perfectionner, de corriger ses erreurs » (p. 15), « ses fautes» (p. 16). Loin

\footnotetext{
${ }^{2}$ Une analyse détaillée de ce roman figure dans Milat (2010).

${ }^{3}$ La mise en scène de l'écriture avec, comme personnages principaux, l'écrivain masculin et son texte, féminin, constitue du reste, dans les romans robbe-grillétiens, un thème récurrent: voir par exemple, sur La Maison de rendez-vous, Janvier (1965) et Payot (1969). Elle s'inscrit bien évidemment dans le droit fil de l'esthétique néo-romanesque telle que Ricardou (1967, p.111) l'a codifiée dans son célèbre chiasme: "Ainsi un roman est-il pour nous moins l'écriture d'une aventure que l'aventure d'une écriture.»
} 
donc de souffrir de ces corrections, l'enfant s'en trouve au contraire rehaussée: «De tout cela-les brûlures, les cinglons, le foutre ensanglanté - [elle] tire une fierté légitime.» (p. 234) Robbe-Grillet admire l'œuvre qu'il vient d'accomplir, comme le père de la jeune fille « ne peut qu'admirer sans réserve [sa] perfection et [sa] grâce » (p. 27). Ayant finalement subi de la part de son père les derniers outrages - ou joui des ultimes hommages -, la jeune fille a perdu sa virginité. Ayant abandonné sa "guêpière noire» $(R S$, p. 13$)$ pour «une robe noire élégante, longue et austère » (p. 235), elle "décide alors de ne plus s'appeler Gigi, qui fait par trop enfantin, mais Gynée, mot par lequel en grec on désigne les jeunes femmes en âge d'être consommées» (p. 234). Effectivement, Un roman sentimental est désormais en mesure d'être consommé par ses lecteurs. Le romancier, «nageant dans l'extrême béatitude» (p. 223), et son œuvre parachevée peuvent donc, à la dernière ligne du roman, déclarer d'une même voix : «Ainsi vivrons-nous à jamais dans les forteresses du ciel. » (p. 253) Tous deux accèdent à l'immortalité littéraire.

\section{Les vertus de la pluralité de sens}

Ainsi, le rouge, le noir et la violence érotique mortifère qui s'exprime au travers de ces deux couleurs constituent pour Robbe-Grillet de simples matériaux, qu'il appelle des «générateurs » (1972a, p. 160) textuels et qu'il emprunte aux stéréotypes de «l'imagerie populaire contemporaine [...]: couvertures illustrées des romans qu'on vend dans les gares, 
affiches géantes, revues pornographiques des sex-shops, publicités vernies des magazines de mode» (p.161). Sur ces matériaux, l'écrivain exerce «le pouvoir de [s]a liberté » (p.161). Il joue avec leurs signifiants, avec leurs polysémies, avec leurs connotations, avec les liens d'analogie qu'ils possèdent avec d'autres matériaux, avec les différents contextes de leurs occurrences, pour finalement produire son texte. Ainsi, par exemple, les images érotiques se placent tout entières au service du romancier, qui leur attribue au travers de son texte un statut et un contenu sémantique nouveaux. C'est pourquoi Robbe-Grillet les compare aux

figures plates d'un jeu de cartes, dépourvues en elles-mêmes de signification comme de valeur, mais auxquelles chaque joueur donnera un sens, le sien, en les disposant dans sa main puis en les abattant sur la table selon sa propre ordonnance, sa propre invention ( $P$, encart, p. 4).

C'est notamment grâce à ce procédé d'écriture que le roman robbe-grillétien accède à la signification: loin du roman conventionnel qui est réduit «à une signification qui lui est extérieure » (Robbe-Grillet, 1963, p. 40) et à un seul sens imposé d'autorité, il comporte une pluralité de sens, créés par l'écriture elle-même. Caractérisé par sa mobilité, le sens des romans de Robbe-Grillet y est donc « pluriel, aléatoire, équivoque, toujours en train de se faire » (Robbe-Grillet, 1972b, p. 175).

Ces sens multiples, c'est au lecteur de les manifester. Son rôle n'est pas « de recevoir tout fait un monde achevé, plein, clos sur lui-même, c'est au contraire de participer à une création, d'inventer à son tour l'œuvre - et le monde - et d'apprendre ainsi à inventer sa propre vie » (Robbe-Grillet, 1963, p. 134). En lisant un roman robbe-grillétien, le lecteur est en effet placé devant un texte qui rompt avec l'horizon d'attente qu'il en a et, partant, il est 
amené à reconsidérer à la fois ses points de repère, ses outils de préhension intellectuelle et ses habitudes d'envisager le monde ainsi que la vie : l'objectif est de libérer le lecteur « de toutes [ses] censures: la morale, la raison, la logique, le respect de l'ordre établi » (Robbe-Grillet, 1987, p. 182).

Les images mêlant le rouge et le noir - Éros et sa violence débouchant sur Thanatos - que Robbe-Grillet place dans ses romans ne visent donc pas une représentation réaliste. En fait, elles ont notamment pour fonction de montrer au lecteur sa face cachée : il s'agit de «tirer au grand jour des images qui se trouvent déjà, de toute façon, dans sa tête, pour les examiner à loisir, donc les démasquer, les mettre en question et apprendre à vivre en bonne entente avec elles, c'est-à-dire à les dominer » (Robbe-Grillet, 1978, p.171). En exhumant et en reprogrammant les pulsions refoulées au plus profond de l'inconscient, le roman remplit donc une fonction cathartique, non seulement pour l'écrivain - «j'écris pour détruire, en les décrivant avec précision, des monstres nocturnes qui menaçaient d'envahir ma vie éveillée » (Robbe-Grillet, 1984a, p. 17) -, mais aussi pour le lecteur, dont l'aliénation a vocation à être transmutée en liberté : le Nouveau Roman a en effet pour ambition de ne créer rien de moins que «l'homme libre futur » (Robbe-Grillet, 1987, p. 82).

\section{Bibliographie}

AlleAU, René. (1953), Aspects de l'alchimie traditionnelle, Paris, Minuit.

AnZIEU, Didier. (1965), « Le Discours de l'obsessionnel dans les romans de Robbe-Grillet», Les Temps Modernes, vol. 21, no 233, octobre, p. 608-637; repris dans Le Corps de l'œuvre, 
Paris, Gallimard, coll. "Connaissance de l'inconscient», 1981, p. 256-281.

Assouline, Pierre. (2007), « Robbe grillé »,

$<$ http://passouline.blog.lemonde.fr/2007/10/15/robbegrille/>, 15 octobre.

BeigBeder, Frédéric. (2007), «Gilles de Rais-Grillet», Lire, novembre, p. 8.

Bonnefoy, Claude. (1978). "Spécial Robbe-Grillet», Les Nouvelles Littéraires, nำ 2660, 9-16 novembre, p. 29.

CANSEliET, Eugène. (1972), L'Alchimie expliquée sur ses textes classiques, Paris, Jean-Jacques Pauvert.

Chevalier Jean et Alain Gherbrant. ([1969] 1982), Dictionnaire des symboles, Paris, Robert Laffont / Jupiter, coll. «Bouquins».

ConTAT, Michel. (2007), «Alain Robbe-Grillet ou les infortunes du pornographe », Le Monde des livres, 26 octobre, p. 2.

DhAEnEns, Jacques. (1970), La Maison de rendez-vous de RobbeGrillet. Pour une philologie sociologique, Paris, Lettres modernes, coll. «Archives des Lettres modernes », no 113.

EliADE, Mircea. (1978), Le Mythe de l'alchimie, Paris, Éditions de L'Herne.

EvolA, Julius. (1988), La Tradition hermétique. Les symboles et la doctrine de l'«art royal» hermétique, Paris, Éditions traditionnelles.

—. (1968), Métaphysique du sexe, Paris, Payot.

FORRESTER, Viviane. (1976), «Robbe-Grillet publie un étrange roman où tout parle », La Quinzaine Littéraire, no 226, $1^{\mathrm{er}}$ 15 février, p. 9. 
FulCANELLI. ([1930] 1965), Les Demeures philosophales et le symbolisme hermétique dans ses rapports avec l'art sacré et l'ésotérisme du grand œuvre, Paris, Jean-Jacques Pauvert.

—. ([1926] 1964), Le Mystère des cathédrales et l'interprétation ésotérique des symboles hermétiques du grand ouvre, Paris, Jean-Jacques Pauvert.

GARCIN, Jérôme. (2007), «Un conte de fées, version Michel Fourniret », Le Nouvel Observateur, no 2243, 1 ${ }^{\mathrm{er}}$ novembre, p. 106.

Goulet, Alain. (1982), Le Parcours mœbien de l'écriture: Le Voyeur, Paris, Lettres modernes, coll. «Archives des Lettres modernes ».

GuÉNON, René. ([1962] 1994), Symboles de la science sacrée, Paris, Gallimard.

HEnRIOT, Émile. (1955) «Le Prix des Critiques: Le Voyeur, d'Alain Robbe-Grillet », Le Monde, 15 juin, p. 9; repris dans Dossier de presse Les Gommes et Le Voyeur d'Alain RobbeGrillet, textes réunis et présentés par Emmanuelle LAMBERT, Paris, Imec-UGE, coll. «10/18 - Dossier de presse », 2005, p. 191-197.

JANVIER, Ludovic. (1965), « Hong-Kong, Alice», Critique, vol. XXI, no 223, décembre, p. 1043-1051.

JUNG, C. G. (1971), Les Racines de la conscience. Études sur l'archétype, Paris, Buchet/Chastel [pour la trad.].

—. ([1944] 1970), Psychologie et alchimie, Paris, Buchet/Chastel [pour la trad.].

LIGER, Baptiste. (2007), «Alain Robbe-Grillet: Rosse Bonbon », L'Express en ligne, $<$ http://livres.lexpress.fr/critique.asp/idC=13141/idR=9/id $\underline{G=>}, 15$ octobre. 
LiNKHORN, Renée. (1976), «L'Amérique dans deux romans français contemporains ", The French-American Review, vol. 1, no 1 , hiver, p. 31-46.

MiLAT, Christian. (2010), «Un roman sentimental: le dernier "nouveau roman" robbe-grillétien? », dans Roger-Michel AllemAND et C. Milat (dir.), Alain Robbe-Grillet : balises pour le XXIe siècle, Actes du colloque international d'Ottawa (1 ${ }^{\mathrm{er}}$ 3 juin 2009), Ottawa / Paris, Presses de l'Université d'Ottawa / Presses Sorbonne Nouvelle, p. 483-491.

-. (2001), Robbe-Grillet, romancier alchimiste, Paris / Ottawa, L'Harmattan / Les Éditions David.

PAYOT, Roger. (1969), "Robbe-Grillet et le Dieu de Leibniz », Preuves, no 219-220, juillet-septembre, p. 107-114.

Ricardou, Jean. (1967), Problèmes du Nouveau Roman, Paris, Seuil, coll. « Tel Quel ».

RoBbe-GriLlET, Alain. (2007), Un roman sentimental, Paris, Fayard, coll. "Littérature générale »; les citations de cet ouvrage sont suivies, entre parenthèses, d'un renvoi aux pages, précédé du sigle $R S$.

—. (1987), Angélique ou l'Enchantement, Paris, Minuit.

—. (1985), cité par Jeanyves Guérin, « Rétrospection. Entretien avec Alain Robbe-Grillet », Esprit, no 101, mai, p. 116-117.

—. (1984a), Le Miroir qui revient, Paris, Minuit.

-. (1984b), "Alain Robbe-Grillet par lui-même», entretien avec Jacques HenRIC, Art Press, no 88, décembre, repris dans Le Voyageur, Paris, Seuil, coll. « Points », 2001, p. 437-448.

-. (1981), Djinn, Paris, Minuit; les citations de cet ouvrage sont suivies, entre parenthèses, d'un renvoi aux pages, précédé du sigle $D$.

—. (1978), " "Histoire de rats" ou La vertu, c'est ce qui mène au crime», Obliques, no 16-17, 1978, p. 169-172; repris dans 
Le Voyageur. Textes, causeries et entretiens (1947-2001), Paris, Christian Bourgois, 2001, p. 121-127.

- (1976), "Robbe-Grillet à la question », dans Jean RiCARDou (dir.), Robbe-Grillet: analyse, théorie, t. II : Cinéma / Roman, p. $410-433$.

-. (1972a), "Sur le choix des générateurs », dans Jean RICARDOU et Françoise van Rossum-GUYON (dir.), Nouveau Roman : hier, aujourd'hui. t. II., Paris, U.G.É., coll. « 10/18», p. 157-162.

-. (1972b), intervention dans la Discussion qui suit la communication de Claude Ollier, "Improvisation et théorie dans la création cinématographique», dans Jacques Bertrand (dir.), Art et science : de la créativité, Paris, U.G.É., coll. «10/18», p. 175.

-. (1970), Projet pour une révolution à New York, Paris, Minuit; les citations de cet ouvrage sont suivies, entre parenthèses, d'un renvoi aux pages, précédé du sigle $P$.

- . (1963), Pour un nouveau roman, Paris, Minuit.

- (1955), Le Voyeur, Paris, Minuit; les citations de cet ouvrage sont suivies, entre parenthèses, d'un renvoi aux pages, précédé du sigle $V$.

SoRIN, Raphaël. (2007), "Robbe-Grillet en vieux dégueulasse », $<$ http://lettres.blogs.liberation.fr/sorin/2007/10/robbegrillet-e.html>, 15 octobre.

StoltzFus, Ben F. (1964), «Camus et Robbe-Grillet: la conscience tragique de L'Étranger et du Voyeur », dans John H. MatThews (ed.), Un Nouveau Roman? Recherches et tradition (La critique étrangère), Paris, Lettres modernes, coll. «La Revue des Lettres modernes », no 94-99, p. 153166. 
SZEBENI, Nicolas Melchior. ([1944] 1970), «Melchior Cibinensis », cité par C. G. Jung, Psychologie et alchimie, Paris, Buchet / Chastel.

VALENTIN, Frère Basile, de l'ordre de Saint-Benoît. (1956), Les Douze Clefs de la philosophie, traduction, introduction, notes et explication des images par Eugène Canseliet, Paris, Minuit.

WEiL-MALHERBE, Rosanne. (1965), « Le Voyeur de Robbe-Grillet : un cas d'épilepsie du type psychomoteur», The French Review, vol. 38, no 4, février, p. 469-476.

\section{Résumé}

La critique a depuis longtemps mis l'accent sur la violence sexuelle qui s'exerce dans les romans d'Alain Robbe-Grillet, mais elle n'a guère produit que des études, en particulier féministes, qui se sont attachées à analyser ce thème d'un point de vue psychologisant, et ce, autant par rapport aux personnages que vis-à-vis de l'auteur lui-même. Le présent article se propose, lui, d'envisager Éros et son corrélat, Thanatos, à travers leurs manifestations chromatiques, le rouge et le noir, et d'un point de vue formel : il s'agira d'analyser les fonctions remplies, au sein de plusieurs romans robbegrillétiens, par différentes occurrences de ces deux pôles, considérés, pour reprendre un concept cher au Nouveau Romancier, en tant que "générateurs " textuels. Cette étude permettra notamment de montrer que ces deux types de "matériaux», loin de répondre à des visées anecdotiques ou ludiques, participent au contraire activement à la production du texte et à la pluralité des sens offerts au lecteur. 


\begin{abstract}
Critics have for a long time focused on the sexual violence perpetrated in the novels of Alain Robbe-Grillet, but they have primarily produced studies, particularly feminist ones, which have analyzed this theme from a psychological point of view, as much in relation to the characters as to the author himself. This article seeks to consider Eros and its correlate, Thanatos, through their chromatic expressions, red and black, and from a formal point of view. It analyzes the functions performed, in several of Robbe-Grillet's novels, by different occurrences of these two poles considered as textual "generators" to borrow a term dear to the New Novelist. The study shows in particular that far from fulfilling trivial or ludic objectives, these two types of "materials" actively participate instead in the text's production and in the multiplicity of meanings offered to the reader.
\end{abstract}

